\title{
Aprovechamiento de los lodos provenientes de plantas de tratamiento de aguas residuales como materia prima en la industria de la construcción: revisión bibliográfica
}

Luis Araujo', Sandra Molina², Leidi Noguera ${ }^{3}$

\section{Resumen}

En los últimos años el campo de la construcción ha tenido un desarrollo acelerado, lo que se ha traducido en un incremento de la cantidad de aguas residuales domésticas y que producto de sus tratamientos se generan lodos a los que se les puede buscar un uso alternativo con el fin de mitigar los impactos ambientales que estos generan. La disposición adecuada de estos lodos está adquiriendo cada vez más relevancia, pues son provenientes de plantas de tratamiento de aguas residuales y poseen características muy diversas, además de específicas; es por esto que a lo largo de los últimos años se han encontrado propuestas concretadas sobre el aprovechamiento de estos biosólidos en el campo de la construcción. Este trabajo se concentra en describir y mostrar el estado de arte en cuanto a los logros alcanzados por diversos estudios realizados en pro de aprovechar de forma acertada los lodos generados en las plantas como materia prima para la fabricación de ladrillos elaborados de arcilla.

Palabras clave: ambientales, arcilla, biosólidos, ladrillos, lodo.

\footnotetext{
IIngeniero de minas, docente del programa Ingeniería de Minas de la Fundación Universitaria del Área Andina sede Valledupar. Correo: laraujodareandina.edu.co

IIngeniera de minas, docente del programa Ingeniería de Minas de la Fundación Universitaria del Área Andina sede Valledupar. Correo: smolina17dareandina.edu.co

${ }^{3}$ Estudiante del programa Ingeniería de Minas de la Fundación Universitaria del Área Andina sede Valledupar. Correo: Inoguera4@ estudiantes.areandina.edu.co
} 


\section{Introducción}

"Los lodos de aguas residuales y de algún proceso industrial son considerados como residuos peligrosos, que demandan tratamiento y manejo especial desde el punto de vista ambiental y sanitario". (Ubaque et al., 2013).

La gestión de lodos es uno de los problemas ambientales más asociados al tratamiento de aguas residuales. La problemática fundamental relacionada con los lodos tiene su origen en que en la mayoría de los casos hay un residuo que gestionar, por tal motivo se hace necesario buscar una alternativa de uso final con el fin de reducir la cantidad generada anualmente en la planta de tratamiento de aguas residuales. Existe la posibilidad de utilizar estos lodos en combinación con las arcillas de cerámicas explotadas por las unidades de producción que brinden la alternativa más viable y acertada en cuanto a especificaciones de combinación.

Los beneficios de utilizar lodos como mezclas para la obtención de ladrillos se evidencian en la disminución de la cantidad de estos en la laguna de sedimentación, como se había mencionado anteriormente; así mismo, se busca disminuir los impactos que se generan por estos lodos, pues haciendo referencia a lo mencionado en el primer párrafo de esta introducción.

En algunos países como Colombia y México se han adelantado estudios en los cuales se evidencia el beneficio del tratamiento y utilización de los lodos productos de tratamientos de aguas residuales en el campo de la elaboración de ladrillos con arcilla, específicamente en el área de la construcción, además existen diversas combinaciones de los lodos con las arcillas para otras aplicaciones. De esa forma, este estudio se centra en el aprovechamiento de los lodos provenientes de plantas de tratamiento de aguas residuales del municipio de Valledupar, con el fin de que puedan ser utilizados para la fabricación de ladrillos.

\section{Antecedentes}

Se tiene a continuación un breve resumen de estudios ordenados por año (primero los más antiguos) realizados para determinar el beneficio y factibilidad de usar los lodos como materia prima en la fabricación de ladrillos en combinación con la arcilla. 
Ortiz, Gutiérrez y Sánchez (1995)

plantearon una propuesta de manejo de los lodos residuales de la planta de tratamiento de la ciudad industrial del Valle de Cuernavaca, Estado de Morelos, México, estos afirman que los lodos producidos diariamente en la planta son aproximadamente en proporción de veinte toneladas y que durante más de quince años se dispusieron en los alrededores de la planta y que actualmente se envían a un relleno sanitario ubicado en el occidente del estado de Morelos, que no cumple con los requisitos de la legislación Mexicana.

Grajales, Monsalve y Castaño (2006)

formularon un programa de manejo integral de los lodos generados en la planta de tratamiento de aguas residuales de la Universidad Tecnológica de Pereira, con el cual se buscó convertir el lodo en un material útil que pudiera ser incorporado dentro de las áreas verdes de la Universidad.

Hernández et al (2006)

evaluaron el uso de lodos aluminosos como agregado en la fabricación de ladrillos cerámicos, aprovechando la composición química de estos lodos estabilizándolos e inmovilizando sus elementos tóxicos, estos autores afirmaron que la composición mineralógica, tamaño de partículas y la plasticidad de los lodos generados por la planta de potabilización de la ciudad de Pereira, eran un material no apto para ser empleados como agregados en la fabricación de ladrillos cerámicos.

Debido a lo anterior, se recomendó sustituir la arena por lodos aluminosos para la fabricación de los ladrillos en valores superiores al 50\%, lo que permitió verificar que al incrementar el porcentaje de lodos la absorción de humedad aumentaría, afectando la resistencia de los ladrillos, mientras que las unidades experimentales que contenían menos del $40 \%$ de lodos aluminosos arrojaban una resistencia, la cual cumplía con lo establecido la Norma Técnica Colombiana NTC 4205 para unidades de mampostería no estructural.

Quinchía, Valencia y Giraldo (2007) realizaron un estudio cuyo foco principal fue el uso de lodos provenientes de la industria papelera en la elaboración de paneles prefabricados para la construcción, este trabajo consistió 
en la elaboración de paneles prefabricados como elementos no estructurales para la construcción, a partir del aprovechamiento de los lodos residuales del tratamiento de las aguas servidas de la industria papelera.

En esta investigación se realizaron tres mezclas en relación con el contenido de yeso, es decir, las diferentes mezclas de yeso y lodo obedecían a un patrón muy ordenado, se observaron 10:90, $15: 85$ y $20: 80$.

La fábrica Cedal S.A. (2012) en Ecuador determinó la viabilidad de utilizar lodos residuales en diversas proporciones y composiciones con el fin de reemplazar porcentualmente las materias primas originales empleadas en la fabricación de ladrillos. Se emplearon los siguientes componentes en las unidades prueba: caolín, arcilla, diatomita y lodo, estos lodos en base seca y húmeda, haciendo variaciones en los porcentajes de lodo entre el $10 \%$ y el $40 \%$ para los ladrillos, mientras que para los adoquines la variación de lodos fue entre el $10 \%$ y el $20 \%$, empleando adicionalmente arena, chispa, piedra y cemento.

Las muestras que se pudieron someter a los ensayos de compresión fueron aquellas que contenían lodo en un por- centaje del 10\%, arrojando una resistencia a la compresión de $46 \mathrm{~kg} / \mathrm{cm} 2$, los cuales, según la norma NTE INEN 297:78, cumplen con los requisitos para ladrillos huecos de tipo E. Con base en los resultados, la empresa recomendó que para la elaboración de ladrillos empleando lodos húmedos estos deben ser sometidos a un proceso de secado con el fin de minimizar el contenido de humedad.

García-Ubaque, García-Vaca y Vaca-Bohórquez (2013)

realizaron un análisis donde se presentaron resultados de un estudio piloto de lixiviación de metales de piezas cerámicas elaborados con mezclas de arcillas y lodos provenientes de la planta de tratamiento de aguas residuales industriales de la planta ensamble de G. G. Colmotores en Bogotá (Colombia), estos autores afirman que con los resultados obtenidos se muestra que existe afinidad entre la arcilla y el tipo de lodo utilizado y que los ladrillos fabricados con mezclas de arcilla y lodo respectivamente 99:1, 95:5, 90:10, 80:20 y 60:40, presentan bajos niveles de lixiviación. Se encuentra que la proporción de mezcla que permite la mayor remoción para todos los 
metales considerados es 95:5 de arcilla y lodo respectivamente.

Camargo (2013) determinó la factibilidad de emplear los lodos residuales de la PTAR del municipio de Chinavita, para esto se desarrollaron análisis agronómicos, contenidos de metales pesados y análisis microbiológicos para ser evaluados y comparados según la norma EPA 40 CFR parte 503, la cual establece alternativas para la estabilización química, empleando cal y digestión anaerobia para lograr la estabilización biológica. Los resultados obtenidos por los análisis desarrollados, establecieron que el posible uso que se le puede dar a estos lodos residuales esta direccionado a emplearse como enmienda orgánica para mejorar propiedades físicas y biológicas de suelos, ya que estos lodos cumplen con los valores permisibles para ser empleados como abonos orgánicos debido a su contenido de metales pesados, $\mathrm{pH}$ y materia orgánica presente en los mismos.

Bermeo e Idrovo (2014) publicaron un estudio titulado Aprovechamiento de lodos deshidratados generados en plantas de tratamiento de agua potable y residual como agregado para materiales de construcción, ellos realizaron análisis complementarios de dicho documento donde analizaron lodos procedentes de las Laguna de Estabilización ubicadas en Ucubamba y parte integrante del sistema de tratamiento de las aguas residuales domesticas de la ciudad de Cuenca-Ecuador, además de los lodos procedentes de las operaciones de potabilización de la Planta de tratamiento de Sústag.

En la Universidad de Medellín, Gutiérrez et al. (2014) tratan un tema bastante interesante como lo es el "tratamiento de lodos generados en el proceso convencional de potabilización de agua":

Estos autores investigaron los lodos aluminosos del proceso de sedimentación, sabiendo que las plantas convencionales incluyen coagulación, floculación, sedimentación y filtración y que además los lodos se producen en todos los procesos, teniendo en cuenta esto determinaron que el mayor porcentaje de lodos del sistema de potabilización estudiado proviene de las unidades de sedimentación.

Salazar (2015) evaluó las propiedades físicas como porcentaje de absorción de agua, porcentaje de contracción longitudinal y pérdida de masa por calcinación de los ladrillos obtenidos de veinte distintas formulaciones. En este 
estudio se decidió que, para cada una de esas formulaciones, se tendría que variar la temperatura de quema de $700^{\circ} \mathrm{C}$ a $1100^{\circ} \mathrm{C}$ y determinaron que el más adecuado correspondió a $1000^{\circ} \mathrm{C}$.

En Ecuador, Cachago y Caguano (2016)

presentaron el trabajo de grado titulado Utilización de lodos de la planta de tratamiento de agua residuales de la empresa Franz Viegener F. V-Área Andina S.A. para la elaboración de ladrillos artesanales, esta investigación basada en la verificación de la posible utilización de lodos residuales como material para la elaboración de ladrillos, dio a conocer la caracterización del ladrillo artesanal convencional utilizado en construcciones y el ladrillo artesanal utilizado con diferentes porcentajes de lodo residual, en variaciones de $25 \%, 50 \%$ y $100 \%$.

Con los resultados obtenidos se concluyó que el tipo de suelo con el cual son elaborados los ladrillos artesanalmente es una arcilla que posee una alta plasticidad, debido a que al realizar el ensayo de compresión simple la dosificación optima que cumplió con la resistencia requerida por la norma fue la dosificación 50:50, 50\% lodo residual, 50\% suelo natural, estos resultados mostraron que ambos tipos de ladrillo se deforman por aplastamiento, mientras que el ensayo a flexión arroja dos tipos de falla, una vertical para los ladrillos elaborados con lodo residual, mientras que los ladrillos convencionales presentan una falla diagonal. Los autores concluyen y recomiendan no emplear estos ladrillos para uso estructural ya que no cumplen con valores límites de corte.

Muñoz (2016),

presentó el estudio titulado "Propuesta para el aprovechamiento de los lodos provenientes de plantas de tratamiento de aguas startwater como materia prima en la fabricación de ladrillo", en este se llevó a cabo la caracterización de los lodos, determinando parámetros de composición química como también de propiedades físicas, se definieron tres mezclas de lodo y arcilla para la fabricación de los ladrillos.

Reinoso (2017),

realizó un estudio titulado "Análisis comparativo de la resistencia a compresión de ladrillos tradicionales y ladrillos elaborados a base de lodos de la planta de tratamiento de agua potable de la red Casigana, como 
sustituto parcial de la arcilla", donde se elaboraron ladrillos tradicionales y ladrillos con lodo de la planta de tratamiento de agua potable red $\mathrm{Ca}$ sigana, como sustituto parcial de la arcilla, en los porcentajes de $5 \%$, $10 \%$ y $15 \%$.

Por otro lado, Fuentes, Isenia y Asencio (2017) publicaron el artículo titulado

"Biosólidos de tratamiento de aguas residuales, como adiciones en la elaboración de ladrillos cerámicos", en este se hizo la recolección de biosólidos de la planta de tratamiento El Salguero del municipio de Valledupar, Cesar, y las arcillas provenientes del municipio de San Juan del Cesar en La Guajira en un sitio de explotación y fabricación de ladrillos artesanales. Los autores concluyeron que la adición de los lodos en la elaboración de los ladrillos no interfiere en la conformación del material durante el proceso de moldeo, prensado y secado.

\section{Conclusiones}

Se pudo observar el gran campo que tiene por recorrer la utilización de los lodos producidos en el proceso de tratamientos de aguas residuales, es así como se pueden especificar o, más bien, como se pueden apuntar a diversas alternativas de uso y de aprovechamiento de tal forma en busca de la reducción de los impactos ambientales que estos pueden traer.

En el campo de la construcción específicamente se observó que ha sido acertada, en algunos casos, la utilización de este residuo como materia prima en combinación con la arcilla, es así entonces como se concluye que los lodos pueden ser una alternativa accesible al momento de elaborar ladrillos de arcilla más el residuo, pues si se analiza detenidamente el resumen de los estudios presentados anteriormente se evidencia que para poder dar con una buena combinación de arcilla-lodo es necesaria la realización de diversos laboratorios.

\section{Referencias}

Bermeo, M. e Idrovo, E. (2014). Aprovechamiento de lodos deshidratados generados en plantas de tratamiento de agua potable y residual como agregado para materiales de construcción.

Cachago Alquinga, M. P. y Caguano Cevallos, C. D. (2016). Utilización de lodos de la planta de tratamiento de agua residual de la 
Empresa Franz Viegener FV Área Andina S.A. para la elaboración de ladrillos artesanales. Tesis de grado, Quito: UCE).

García Ubaque, C., García Vaca, M. y Vaca Bohórquez, M. (2013). Encapsulamiento de lodos de plantas de tratamiento de aguas residuales de la industria automotriz en matrices de arcilla. Tecnura, 17(38).

Grajales, S., Monsalve, J. y Castaño, J. (2006). Programa de Manejo Integral de los lodos generados en la planta de tratamiento de aguas residuales de la Universidad Tecnológica de Pereira. Scientia et Technica, 2(31).

Hernández, D., Villegas, J., Castaño, J. y Paredes, D. (2006). Aprovechamiento de lodos aluminosos generados en sistemas de potabilización, mediante su incorporación como agregado en materiales de construcción. Revista Ingenierías Universidad de Medellín, 5(8).

Molina, N., León, S. y Mendoza, J. (2017). Biosólidos de tratamiento de aguas residuales domésticas, como adiciones en la elaboración de ladrillos cerámicos. Producción+Limpia, 12(2).

Muñoz, M. (2016). Propuesta para el aprovechamiento de los lodos provenientes de plantas de tratamiento de aguas startwater como materia prima en la fabricación de ladrillo. Tesis de grado, Fundación Universidad de América, Bogotá, D. C.

Quinchía, A., Valencia, M. y Giraldo, J. (2007). Uso de lodos provenientes de la industria papelera en la elaboración de paneles prefabricados para la construcción. Revista EIA, 8, 9-19.

Peralta Pintado, J. R. (2018). Elaboración de ladrillos cerámicos utilizando lodos generados en la planta de tratamiento de agua potable de Tixán en la ciudad de Cuenca. Tesis de maestría, Ecuador.

Salazar Carrera, P. J. (2015). Fabricación de ladrillos cerámicos utilizando como aditivos rotura y lodos de la planta de tratamiento de agua de la empresa Edesa S.A. Tesis de grado, Universidad Central del Ecuador, Quito. 